\title{
Epidemiología de la prematuridad, sus determinantes y prevención del parto prematuro
}

\author{
Luis Alfonso Mendoza Tascón ${ }^{1,2}$, Diana Isabel Claros Benítez ${ }^{2}$, Laura Isabel Mendoza \\ Tascón ${ }^{2}$, Martha Deyfilia Arias Guatibonza ${ }^{1}$, Claudia Bibiana Peñaranda Ospina. 1,2 \\ ${ }^{1}$ Fundación Hospital San José de Buga. ${ }^{2}$ Facultad Ciencias de la Salud, Programa de Medicina, Unidad Central del Valle \\ del Cauca. Tuluá. Colombia.
}

\section{RESUMEN}

La prematuridad es un grave de problema de salud pública por la gran morbilidad y mortalidad que generan, además, de los elevados costos económicos y sociales que ocasiona su atención. A nivel mundial, aproximadamente uno de cada diez neonatos nacen prematuros. Sus determinantes son múltiples. En el parto prematuro están involucrados además de los determinantes biológicos, los que son responsabilidad del sector salud y los que son responsabilidad del estado, como son los determinantes políticos, ambientales, sociales y económicos. Es por ello que la prevención y el tratamiento de la prematuridad debe ser una política pública obligada para todas las naciones, e involucra a muchos actores. Las estrategias empleadas para prevenir y tratar al parto prematuro son amplias y van desde los cuidados preconcepcionales, hasta la atención del parto y del neonato en el periodo postnatal.

\section{PALABRAS CLAVE: Prematuridad, determinante de la salud, morbilidad y mortalidad neonatal}

\section{SUMMARY}

Prematurity is a serious public health problem by the high morbidity and mortality also generated high economic and social costs caused by its staff. Globally, about one in ten infants born prematurely. Its determinants are numerous. In preterm birth are involved in addition to biological determinants, which are the responsibility of the health sector and which are the responsibility of the state, such as political, environmental, social and economic determinants. That is why prevention and treatment of prematurity should be a public policy required for all nations, and involves many actors. The strategies used to prevent and treat premature birth are spacious and range from preconception care, to care delivery and newborn in the postnatal period.

KEY WORDS: Prematurity, determinant of health, neonatal morbidity and mortality

\section{INTRODUCCIÓN}

Las circunstancias en que las personas viven y trabajan, afectan la salud de los individuos. Se estima que los determinantes sociales y ambientales tienen dos veces el impacto en la calidad de la atención en salud y en la salud en general de un individuo, que los otros determinantes (1).

Son varios los determinantes que pueden afectar el embarazo en adolescentes y adultas, como, pobreza, estructura familiar, calidad de la vivienda, acceso a alimentos saludables, acceso a servicios 
de salud y de atención primaria, tecnología en salud, cohesión social, percepción de discriminación/ inequidad, acceso a empleo, situación laboral, políticas que apoyen la promoción de la salud, entornos escolares seguros e ingreso a la educación superior (2).

La prematuridad es uno de los resultados de estos determinantes de la salud, el cual se convierte además, en un determinante importante de mortalidad y morbilidad neonatal, con consecuencias a largo plazo (3-5). Los niños que nacen prematuros no sólo tienen mayor mortalidad y morbilidad en el periodo neonatal, si no en etapas posteriores de la vida, resultando en enormes costos $(6,7)$, de ahí que sea concebida como de alta prioridad para la salud pública en algunos países.

\section{DEFINICIONES}

- Prematuridad: Es definido por la Organización Mundial de la Salud (OMS) como el nacimiento que ocurre antes de completarse las 37 semanas o antes de 259 días de gestación, desde el primer día del último periodo menstrual $(8,9)$. Se subdivide en extremadamente prematuros <28 semanas $(5,2 \%$ de todos los <37 semanas; IC 95\%: $5,1 \%-5,3 \%$ ), muy prematuros $28-31$ semanas $(10,4 \%$ de todos los <37 semanas; IC 95\%: 10,3\%-10,5\%) y moderadamente prematuros $32-36$ semanas $(84,3 \%$ de todos los <37 semanas; IC 95\%: $84,1 \%-84,5 \%$ ). Entre estos moderadamente prematuros, se ha clasificado a un subgrupo denominado, "prematuros tardíos" (34-36 semanas) (10,11).

- Determinante de la salud: Son un conjunto de factores personales, sociales, económicos y ambientales que determinan el estado de salud de los individuos o poblaciones. Estos se pueden dividir en dos grupos: 1 . Aquellos que son de responsabilidad multisectorial del estado y son los determinantes económicos, sociales y políticos; 2 . Los que son de responsabilidad del sector salud, dentro del accionar del estado, en beneficio de la salud de la población, en lo que se refiere a vigilancia y control en unos casos, y de promoción y actuación directa en otros. Estos incluyen: a. Aquellos relacionados con los estilos de vida y conductas de salud (toma de decisiones y hábitos de vida, drogas, sedentarismo, alimentación, estrés, conducción peligrosa, mala utilización de los servicios sanitarios, etc.); b. Factores del medio ambiente (físico, químico, biológico, psicosocial, cultural, etc.); c. Factores relacionados con los aspectos biológicos y genéticos (herencia, maduración, envejecimiento, sistemas internos complejos, etc.); d. Los relacionados con los sistemas de asistencia sanitaria (prevención, curación, rehabilitación, accesibilidad a servicios de salud, mala utilización de los recursos, etc.) $(12,13)$.

\section{MAGNITUD DEL PROBLEMA}

Prevalencia de la prematuridad: Para el año 2005, se estimó que $9,6 \%$ (cerca de 12,9 millones) de todos los nacimientos fueron prematuros. Aproximadamente $85 \%$ de esta carga se concentró en África y Asia, donde 10,9 millones de nacimientos fueron prematuros. Alrededor de 0,5 millones se dieron en Europa y el mismo número en América del Norte, mientras que 0,9 millones se produjeron en América Latina y El Caribe (ALC). Las tasas más elevadas se produjeron en África con $11,9 \%$ y América del Norte con 10,6\%. En Europa fue de $6,2 \%$, con la tasa más baja (9). En los Estados Unidos de Norteamérica (EUA) los partos prematuros representan el $12,5 \%$ de todos los nacimientos, es decir, cada minuto nace un prematuro en ese país (14). Para algunos países de ALC en 2010, la situación es similar (Tabla I) (15).

Tabla I

\section{NACIMIENTOS PREMATUROS POR CADA 100 NACIMIENTOS}

\begin{tabular}{lc}
\hline País & \% nacimientos prematuros \\
\hline Costa Rica & 13,6 \\
El Salvador & 12,8 \\
Honduras & 12,2 \\
Belice & 10,4 \\
Uruguay & 10,1 \\
Nicaragua & 9,3 \\
Brasil & 9,2 \\
Bolivia & 9,0 \\
Colombia & 8,8 \\
Panamá & 8,1 \\
Venezuela & 8,1 \\
Argentina & 8,0 \\
Paraguay & 7,8 \\
Guatemala & 7,7 \\
Perú & 7,3 \\
México & 7,3 \\
Chile & 7,1 \\
Cuba & 6,4 \\
Ecuador & 5,1
\end{tabular}

Morbilidad: Los niños que nacen prematuramente tienen mayores complicaciones a corto, mediano y largo plazo. Estos se ven afectados por 
diferentes complicaciones y morbilidades asociadas al parto prematuro y que se extienden a través de los años de vida, afectando la niñez y la etapa adulta, resultando en enormes costos físicos, psicológicos y económicos (Tabla II) $(6,7,11)$. Las estimaciones indican que en 2005 los costos para EUA solamente en términos de gastos médicos, educativos y pérdida de productividad asociada al parto prematuro, fueron superiores a US\$26,2 mil millones (16). Para este mismo año, los promedios de los costos médicos del primer año de atención hospitalaria y ambulatoria, eran cerca de 10 veces mayor para prematuros (US\$32.325) que para neonato a término (US\$ 3.325). La estancia media fue 9 veces más larga para un neonato prematuro (13 días), que uno a término (1,5 días) (11).

\section{IMPACTO A LARGO TÉRMINO DE LOS SOBREVIVIENTES PREMATUROS}

\begin{tabular}{|c|c|c|c|}
\hline \multicolumn{2}{|c|}{$\begin{array}{c}\text { Impacto a largo término de los sobrevivientes } \\
\text { prematuros }\end{array}$} & \multirow{2}{*}{$\begin{array}{l}\text { Compromisos } \\
\text { Ceguera o alta miopía } \\
\text { después de la retinopa- } \\
\text { tía de la prematuridad. } \\
\text { Hipermetropía }\end{array}$} & \multirow{2}{*}{$\begin{array}{l}\begin{array}{l}\text { Frecuencia en } \\
\text { sobrevivientes }\end{array} \\
25 \% \text { de todos los } \\
\text { prematuros extremos. } \\
\text { También afecta a otros } \\
\text { prematuros } \\
\text { pobremente } \\
\text { monitorizados en las } \\
\text { terapias de oxígeno. }\end{array}$} \\
\hline $\begin{array}{l}\text { Resultado a largo } \\
\text { término }\end{array}$ & Deterioro visual & & \\
\hline & Deterioro auditivo & $\begin{array}{l}\text { Pérdida auditiva de } \\
\text { diferente grado }\end{array}$ & $\begin{array}{l}5-10 \% \text { de prematuros } \\
\text { extremos. }\end{array}$ \\
\hline & $\begin{array}{l}\text { Enfermedad pulmonar } \\
\text { crónica de la } \\
\text { prematuridad }\end{array}$ & $\begin{array}{l}\text { Alta o moderada } \\
\text { dependencia al } \\
\text { oxígeno en casa. }\end{array}$ & $\begin{array}{l}40 \% \text { de prematuros } \\
\text { extremos. }\end{array}$ \\
\hline & $\begin{array}{l}\text { Enfermedad } \\
\text { cardiovascular }\end{array}$ & $\begin{array}{l}\text { Incremento de la } \\
\text { presión arterial; } \\
\text { reducción de la función } \\
\text { pulmonar; incremento } \\
\text { de los índices de asma; } \\
\text { falla en el crecimiento } \\
\text { en la infancia, ganancia } \\
\text { de peso acelerada en } \\
\text { la adolescencia. }\end{array}$ & $\begin{array}{l}\text { Pendiente determinar la } \\
\text { magnitud de la carga. }\end{array}$ \\
\hline \multirow[t]{3}{*}{$\begin{array}{l}\text { Neurodesarrollo/efec- } \\
\text { tos conductuales }\end{array}$} & $\begin{array}{l}\text { Trastornos de } \\
\text { ejecución de la } \\
\text { marcha leve }\end{array}$ & $\begin{array}{l}\text { Impedimentos } \\
\text { específicos del } \\
\text { aprendizaje, dislexia, } \\
\text { disminución de logros } \\
\text { académicos. }\end{array}$ & $\begin{array}{l}\text { Pendiente determinar la } \\
\text { magnitud de la carga }\end{array}$ \\
\hline & $\begin{array}{l}\text { Moderado a severo } \\
\text { retardo global del } \\
\text { desarrollo. }\end{array}$ & $\begin{array}{l}\text { Deterioro cognitivo } \\
\text { moderado a severo; } \\
\text { deterioro motor y } \\
\text { parálisis cerebral. }\end{array}$ & $\begin{array}{l}\text { Dependientes de la } \\
\text { edad gestacional y } \\
\text { calidad de los cuidados } \\
\text { neonatales. }\end{array}$ \\
\hline & $\begin{array}{l}\text { Secuelas psiquiátricas } \\
\text { y conductuales }\end{array}$ & $\begin{array}{l}\text { Desorden de } \\
\text { hiperactividad y déficit } \\
\text { de atención; aumento } \\
\text { de trastorno de } \\
\text { ansiedad y depresión. }\end{array}$ & $\begin{array}{l}\text { Pendiente determinar la } \\
\text { magnitud de la carga }\end{array}$ \\
\hline $\begin{array}{l}\text { Efectos familiares, } \\
\text { económicos y sociales }\end{array}$ & $\begin{array}{l}\text { Impacto sobre la } \\
\text { familia; impacto sobre } \\
\text { los servicios de salud; } \\
\text { intergeneracional }\end{array}$ & $\begin{array}{l}\text { Compromiso } \\
\text { psicosocial, emocional } \\
\text { y económico; costos } \\
\text { de la atención en salud; } \\
\text { riesgo de parto } \\
\text { prematuro en la } \\
\text { descendencia }\end{array}$ & $\begin{array}{l}\text { Variación de acuerdo a } \\
\text { los riesgos, factores de } \\
\text { la atención, } \\
\text { discapacidad y } \\
\text { estado } \\
\text { socioeconómico }\end{array}$ \\
\hline
\end{tabular}


Mortalidad: De todas las muertes neonatales en la primera semana de vida y que no se relacionan con malformaciones congénitas, $28 \%$ están asociadas a prematuridad (17). En la mayoría de países de ingresos medios y altos del mundo, la prematuridad es la principal causa de muerte infantil (18). Un $75-80 \%$ de las muertes perinatales ocurren en $<37$ semanas de edad gestacional (19-21) y aproximadamente $40 \%$ de estas muertes ocurren en $<32$ semanas (22), siendo la sepsis y otras enfermedades infecciosas las principales causas de muerte neonatal (23). Las complicaciones secundarias al parto prematuro, son la causa directa más importante de mortalidad neonatal, siendo responsable de $35 \%$ del total de las muertes a nivel mundial, con 3,1 millones de muertes al año y la segunda causa de muerte más común en $<5$ años después de la neumonía (18).

\section{DETERMINANTES DEL PARTO PREMATURO}

Las causas del parto prematuro se pueden dividir en dos subgrupos: 1. Parto prematuro espontáneo: Inicio espontáneo del trabajo de parto o después de la ruptura prematura de membranas ovulares (RPMO). 2. Parto prematuro indicado por un proveedor de salud: Inducción del trabajo de parto o parto por cesárea antes de las 37 semanas de gestación completas por indicaciones maternas, obstétricas o fetales (24).

Determinantes del parto prematuro espontáneo. Este es de origen multifactorial, resultado de la interacción de varios factores que activan la contractilidad uterina antes de que se completen las 37 semanas de gestación.

La RPMO en embarazos simples, es responsable de $8,5-51,2 \%$ y el trabajo de parto espontáneo de $27,9 \%-65,4 \%$ de todos los partos prematuros. Para EUA, 69\% de los partos prematuros fueron espontáneos (25). Sin embargo, estos factores varían según la edad gestacional (26) y según factores sociales y ambientales, pero en la mayoría de los casos, las causas permanecen sin identificar (27).

Varios factores han sido implicados, entre ellos el antecedente de parto prematuro previo, (28) la edad de la madre (<20 años o >35 años), el periodo intergenésico corto y un índice de masa corporal bajo $(29,30)$. Un factor importante asociado al parto prematuro es el embarazo múltiple, que aumenta el riesgo de parto prematuro en casi 10 veces, cuando se le compara con embarazos simples (31).

El embarazo múltiple varía entre los grupos étnicos ( 1 de cada 40 mujeres de África Occidental a 1 en 200 mujeres en Japón), aumenta a medida que la gestante es de mayor edad e incrementa con el aumento de la concepción asistida (32).
Las patologías infecciosas en la madre, juegan un papel importante en la ocurrencia del parto prematuro, incluyendo, infección del tracto urinario, vaginosis bacteriana, sífilis, infección por $\mathrm{VIH}$, corioamnionitis, colonización materna por Strepcococcus del grupo $\mathrm{B}$, entre otros (33-35).

La insuficiencia de cuello uterino se ha asociado a infección uterina ascendente. (36). Otros factores asociados con el aumento de riesgo de parto prematuro son el tabaquismo y consumo de alcohol en exceso, así como la enfermedad periodontal (37). La enfermedad periodontal, en especial la periodontitis apical crónica, se ha encontrado asociada al parto prematuro de muy bajo peso (ORa 3,52; IC 95\%: 1,01-12,32) (38).

Se discute el papel que juega la etnicidad, pero esta se ve influenciada por factores socioeconómicos y estilos de vida, así como por la genética (39). Las anomalías congénitas son otra causa importante de parto prematuro espontáneo, mientras la prematuridad ocurre con mayor frecuencia en varones (40).

Determinantes del parto prematuro indicado por el proveedor de salud. Entre los embarazos simples, los partos indicados representan 20\%$38,3 \%$ de todos los nacimientos. Entre los embarazos simples en EUA, $31 \%$ de los nacimientos prematuros son el resultado de una indicación o decisión médica (del trabajo de parto o de la cesárea) (25).

Barros y cols (41), describen que los 12 grupos de nacimiento prematuros según las condiciones maternas, fetales o placentarias en orden de frecuencia son: causa desconocida (30\%), preeclampsia $(11,8 \%)$, embarazo múltiple $(10,4 \%)$, infección extrauterina $(7,7 \%)$, corioamnionitis $(7,6 \%)$, sangrado de segundo y tercer trimestre del embarazo $(6,2 \%)$, sospecha de restricción de crecimiento intrauterino (RCIU) $(5,8 \%)$, sepsis perinatal $(5,5 \%)$, sangrado temprano (4,8\%), muerte fetal anteparto $(3,7 \%)$, sufrimiento fetal $(3,4 \%)$ y condición materna grave $(3,1 \%)$. Otras causas incluyen, rotura uterina, colestasis, enfermedades maternas de origen renal, hipertensión arterial crónica, obesidad, anemia, asma, enfermedad tiroidea, desnutrición, deficiencia de micronutrientes, diabetes crónica y gestacional, depresión y violencia contra la mujer $(11,39,42,43)$. Los tratamientos de fertilidad aumentan tanto el parto prematuro espontáneo, como el indicado por los proveedores de salud $(42,43)$.

Ananth y cols (39), describen entre neonatos $<35$ semanas, que la preeclampsia sola, se constituyó en la principal causa de parto prematuro indicado con $10,4 \%$. Sin embargo, esta se puede asociar a otras condiciones e indicar el nacimiento antes de las 37 semanas, como a RCIU (5,6\%), sufrimiento fetal $(3,1 \%)$, RCIU y sufrimiento fetal 
(2,6\%), desprendimiento prematuro de placenta $(0,8 \%)$, y a diferentes combinaciones de estas $(0,2-$ $0,5 \%$ ). El sufrimiento fetal solo, indicó el nacimiento prematuro en $11,4 \%$. Este sufrimiento fetal se puede asociar a otras complicaciones e indicar el nacimiento prematuro, como cuando se asocia a RCIU $(3,3 \%)$, desprendimiento prematuro de placenta $(1,6 \%)$ y RCIU con desprendimiento prematuro de placenta $(0,5 \%)$. La RCIU sola, indicó el nacimiento prematuro en $5 \%$, y cuando este se asoció a desprendimiento prematuro de placenta ocasionó el $1,4 \%$ de los partos prematuros indicados. El desprendimiento prematuro de placenta solo, indica el $6,8 \%$ de todos los partos prematuros.

Determinantes sociales del parto prematuro. Desde la declaración de Alma-Ata, las naciones y los actores internacionales, se han comprometido, e incluso abogado por "una salud para todos" en los últimos años (44). A pesar de la mejoría en algunos sectores de la salud, persisten algunos indicadores de inequidad en salud en los sectores de la sociedad más desfavorecidos (45), donde millones de personas llevan niveles de vida deficientes e incluso se ven expuestas a riesgos totalmente prevenibles $(44,45,46)$. Datos de 86 países de bajos y medianos ingresos, informan que estas inequidades persisten y afectan principalmente a los grupos más vulnerables, incluyendo mujeres y niños. En salud, las mayores brechas detectadas son la atención médica especializada (diferencia de un $80 \%$ entre la población más rica y más pobre), inadecuada atención prenatal con un mínimo de 4 visitas a los centros de salud durante el embarazo (diferencia del $25 \%$ entre los subgrupos más y los menos educados), además de las inequidades en la adopción de métodos de planificación familiar (45).

Varias categorias de determinantes afectan la salud. Los atributos de la enfermedad que son influenciados por la posición social (estado financiero, marginalidad social, desempleo a largo plazo, entorno físico, entorno laboral, etc.); factores que pueden alterar las consecuencias de la enfermedad en términos de supervivencia, capacidad funcional y calidad de vida (naturaleza de la enfermedad/lesión, desigualdad en la utilización de los servicios de salud, ausencia de personal de salud, limitaciones logísticas, etc.) (45-48); deficiencia de servicios de salud en sectores de difícil acceso geográfico, como áreas rurales, donde hay ausencia de profesionales de la salud e insuficiente suministro continuo de insumos, incluyendo medicamentos (44-46). Un estudio realizado en Escocia, empleando el "Scottish Index of Multiple Deprivation, "Carstairs index" y "Social Class Based on Occupation", identificó que las tasas de embarazo adolescente afectaron principalmente a la población más desfavorecida desde los determinantes socioeconómicos (49).

El acceso a la anticoncepción en adolescentes y mujeres adultas, se ha identificado como una de las medidas protectoras contra el parto prematuro. Rodriguez y cols (50), evaluaron el impacto de la cobertura y uso de anticonceptivos dentro de los 18 meses posteriores a un nacimiento en la prevención del parto prematuro. Estos autores encontraron que, dentro de la cohorte evaluada, 9,75\% de partos fueron prematuros. Identificaron que la cobertura de anticonceptivos resultó ser protectora contra los nacimientos antes de la semana 37. Por cada mes de cobertura de anticonceptivos, las probabilidades de un parto prematuro disminuyeron 1,1\% (OR 0,989; IC95\%: 0,986-0,993).

El control prenatal (CPN) adecuado se ha identificado como medida protectora contra el parto prematuro. Las últimas recomendaciones de organismos internacionales, como la OMS y National Institute for Health and Clinical Excellence $(51,52)$, dan más importancia a otros indicadores de calidad de la atención prenatal, que al número de visitas durante este, con al menos seis visitas considerado como óptimo. Esto surge del hecho de que no han encontrado diferencias estadísticamente significativas respecto a la morbilidad neonatal y materna, cuando se han completado más de seis visitas, siempre y cuando los demás indicadores de calidad se cumplan (es decir, primera visita antes de la semana 12, suplementos de ácido fólico antes de la semana 8 y los análisis de sangre correspondientes) $(53,54)$. El estudio realizado por Paz-Zuleta y cols (55), describe que el pobre cumplimiento de la atención prenatal es el principal factor de riesgo independiente asociado al parto prematuro (ORa 17,05; IC 95\%: 3,92-74,24) y bajo peso al nacer (ORa 6,25; IC 95\%: 1,28-30,46). Los factores de riesgo social fueron importantes de manera independiente para el bajo peso al nacer (ORa 5,42; IC 95\%: 1,58-18,62). Mendoza y cols (56), describen que la adolescencia (ORa 1,85; IC 95\%: 1,44-2,39), escolaridad <11 años (ORa 1,39; IC 95\%: 1,14-1,71), carencia de marido o compañero estable (ORa 2,20; IC 95\%: 1,74-2,79), carencia de seguridad social en salud (ORa 2,76; IC 95\%: 1,95-3,90) y gravidez $\geq 2$ (ORa 1,70; IC 95\%: 1,38-2,14), fueron los determinantes asociados a una mala adherencia al CPN. Esta mala adherencia al CPN se asoció a prematuridad y/o bajo peso al nacer (ORa 1,66; IC 95\%: 1,332,06) y a mortalidad neonatal (ORa 2,81; IC $95 \%$ : 1,47-5,38). Redding y cols (57), evaluaron los resultados de un programa de acceso a salud comunitaria, utilizando agentes de salud comunitarios, para identificar a mujeres en riesgo de tener pobres resultados en el parto, conectarlos a los servicios de salud y servicios sociales para su 
atención y seguimiento. Encontraron una reducción del riesgo de nacimientos de bajo peso en el grupo intervenido por los agentes comunitarios $(6,1 \%$ vs $13 \%)$, convirtiéndola en una medida protectora (ORa 0,35; IC 95\%: 0,12-0,96).

El desplazamiento y la migración afectan principalmente a comunidades en situaciones socioeconómicas pobres y de violencia. $Y$ son estas poblaciones, donde existe un mayor riesgo de inequidades entre los diferentes determinantes sociales, lo cual coloca a las mujeres, principalmente a las gestantes, en posiciones de riesgo social y vulnerabilidad, (58-61) afectando los resultados neonatales (62-64).

\section{PREVENCIÓN DEL PARTO PREMATURO}

La reducción de los nacimientos prematuros y la mejora de la supervivencia infantil son objetivos ambiciosos. Para lograr estos objetivos, se requiere de la colaboración y coordinación entre gobiernos, donantes nacionales y locales, Naciones Unidas y otros organismos multilaterales, sociedad civil, comunidad empresarial, profesionales e investigadores, todos trabajando juntos para avanzar en la inversión, implementación, innovación e intercambio de información (Tabla III) (11).

Hay nuevos objetivos que buscan reducir las tasas de mortalidad debidas a complicaciones del parto prematuro. Para los países con un nivel de tasa de mortalidad neonatal actual $\geq 5$ por cada 1.000 nacidos vivos, la meta es reducir la mortalidad por nacimientos prematuros en un $50 \%$ entre 2010 y 2025. Para los países con un nivel donde la tasa de mortalidad neonatal actual $<5$ por cada 1.000 nacidos vivos, el objetivo es eliminar las muertes prevenibles de prematuros, centrándose en la atención equitativa para todos y la calidad de la atención, para minimizar a largo plazo la discapacidad (11). La reducción de la carga generada por el parto prematuro tiene una doble vía: La prevención y la atención (11).

\section{Reducción del parto prematuro}

Prevención del parto prematuro: Las intervenciones con efecto probado para la prevención del parto prematuro se agrupan en el periodo preconcepcional y en la atención prenatal.

Cuidados preconcepcionales. Brindan atención a las mujeres y parejas antes y entre las gestaciones (Tablas IV, V, VI) (11).
Intervenciones de la atención prenatal. Existen un conjunto de intervenciones prioritarias e intervenciones basadas en la evidencia durante el embarazo, cuya finalidad es reducir las tasas de nacimientos prematuros y mejorar los resultados neonatales (Tablas VII, VIII) (11).

\section{Reducción de la mortalidad en neonatos prema- turos}

Atención del parto prematuro: Incluye el empleo de tocolíticos, corticosterioides prenatales, antibióticos para la ruptura prematura y prolongada de membranas ovulares, corioamnionitis y otras infecciones en la madre (11).

Atención del neonato prematuro: Incorpora una adecuada reanimación neonatal; atención del neonato en una unidad neonatal integral cuando sea requerido; soporte nutricional y apoyo especial a la alimentación, lactancia materna exclusiva; utilización del Método Madre Canguro para neonatos de peso al nacer <2000 g; cuidados del cordón umbilical con clorhexidina y cuidados de la piel; manejo seguro del soporte de oxígeno; diagnóstico precoz y tratamiento oportuno y adecuado de la hiperbilirrubinemia; atención a las complicaciones del prematuro en especial las respiratorias y las infecciosas, entre otras (11).

\section{CONCLUSIONES}

La prematuridad es el resultado de una compleja red de determinantes que interactúan. Para su prevención, no basta solo las medidas de salud, se deben elaborar políticas y planes de acción integral para minimizar la desigualdad en salud, especialmente entre las mujeres, los neonatos y diferentes subgrupos de niños. Si bien es cierto, que las políticas en salud por sí solas no ocasionan cambios individuales, si pueden proporcionar soluciones para hacer frente a algunos mecanismos que facilitan las inequidades en salud. De otro lado, se deben realizar intervenciones dirigidas a las mujeres en edad reproductiva, atendiendo sus necesidades en salud sexual y reproductiva, incluyendo educación sexual, uso de anticonceptivos, prevención de embarazos en la adolescencia temprana, prevención de embarazos no deseados, atención prenatal de calidad, cumplimiento del enfoque de riesgo para las gestantes e inclusión de personal calificado en la atención del parto. La prevención y tratamiento de la prematuridad, es una de las estrategias más importantes tenidas en cuenta para reducir la mortalidad neonatal e infantil. 


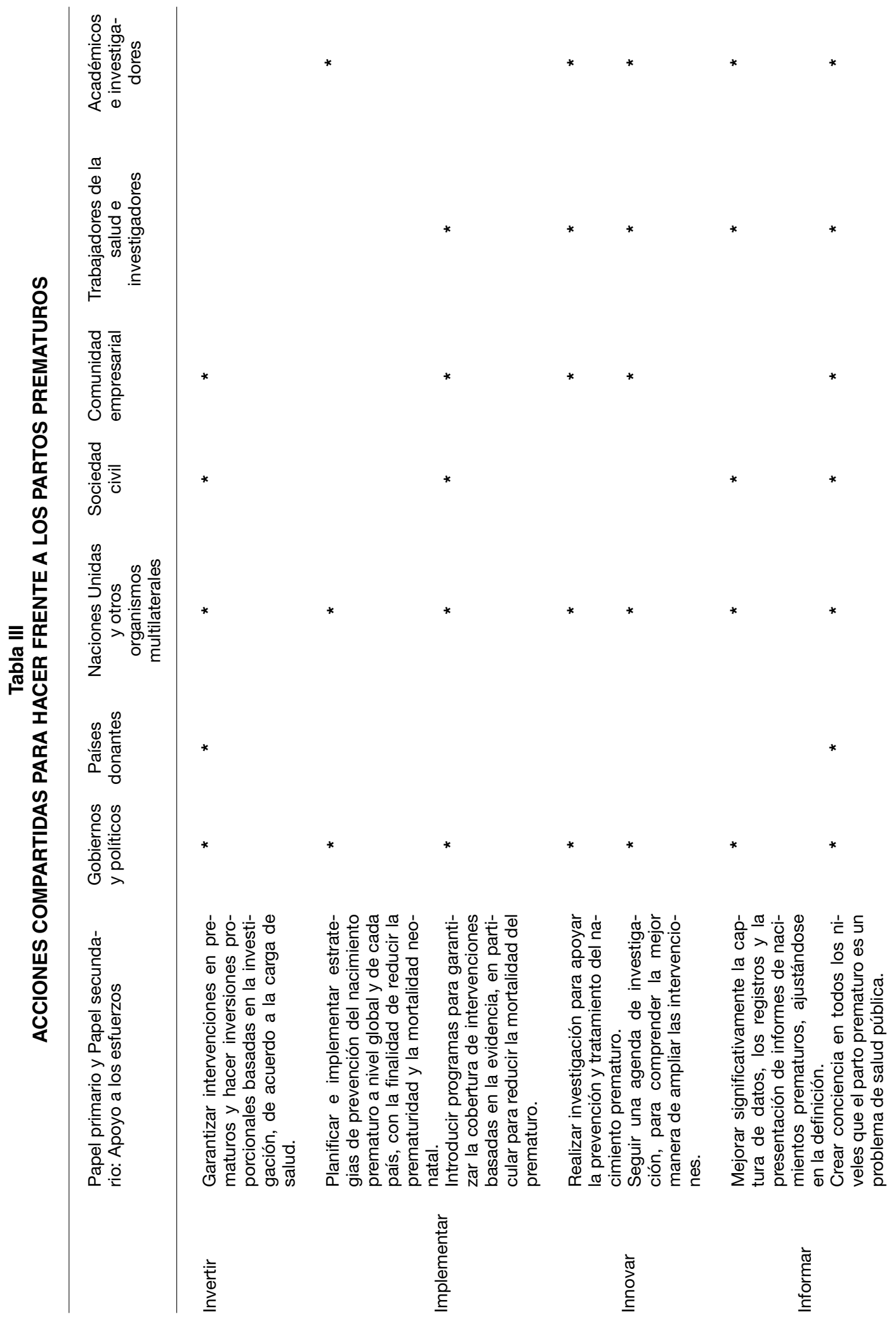




\section{MARCO CONCEPTUAL PARA EL CUIDADO PRECONCEPCIONAL}

\begin{tabular}{lll}
\hline Tipo de intervención & Características & Intervenciones \\
\hline Inmediata & Factores de riesgo biomédico y & Planificación reproductiva. \\
& estilos de vida & Espaciamiento de los nacimientos. \\
& Dieta santiconceptivos. actividad física, suplementación con \\
& micronutrientes. \\
& Detección y manejo de enfermedades crónicas. \\
& Inmunización, detección y tratamiento de enfer- \\
& medades infecciosas. \\
& Optimización de la salud psicológica. \\
& Prevención y tratamiento de abuso de sustancias \\
& como tabaco, alcohol y drogas psicoactivas.
\end{tabular}

\begin{tabular}{lll}
\hline Intermedias & $\begin{array}{l}\text { Familia, estructuras comunitarias } \\
\text { formales y no formales }\end{array}$ & $\begin{array}{l}\text { Servicios de salud esenciales. } \\
\text { Cuidados para niñas, adolescentes y mujeres. } \\
\text { Adecuada nutrición. }\end{array}$ \\
\hline Subyacente & $\begin{array}{l}\text { Entorno físico, social, contexto } \\
\text { económico y político }\end{array}$ & $\begin{array}{l}\text { Medio ambiente saludable. } \\
\text { Empoderamiento de la mujer. } \\
\end{array}$ \\
& $\begin{array}{l}\text { Mujeres con independencia financiera. } \\
\text { Educación femenina. } \\
\end{array}$ & $\begin{array}{l}\text { Prevención de la violencia contra niñas, adoles- } \\
\text { centes y mujeres adultas. }\end{array}$
\end{tabular}

Tabla V

\section{INTERVENCIONES PRIORITARIAS DURANTE EL PERÍODO PRECONCEPCIONAL PARA REDUCIR LAS TASAS DE NACIMIENTOS PREMATUROS}

Servicios de cuidado preconcepcional para la prevención del parto prematuro para todas las mujeres

- Prevenir el embarazo en la adolescencia.

- Prevenir los embarazos no deseados y promover el espaciamiento de los nacimientos y embarazos no planeados.

- Optimizar el peso antes del embarazo.

- Promover una nutrición saludable, incluyendo la administración de suplementos/fortificación de alimentos con micronutrientes esenciales.

- Promover la vacunación de niños y adolescentes.

Servicios de cuidado preconcepcional para las mujeres con factores de riesgo especiales que aumentan el riesgo de parto prematuro

- Tamización para diagnosticar y tratar trastornos de la salud mental y prevenir la violencia en la pareja.

- Prevenir y tratar las infecciones de transmisión sexual, incluido el VIH/SIDA.

- Promover el abandono del consumo de tabaco y limitar la exposición al humo de segunda mano.

- Tamización para diagnosticar y tratar las enfermedades crónicas, como la diabetes y la hipertensión arterial. 


\title{
Tabla VI \\ ACCIONES ANTES Y DURANTE EL EMBARAZO PARA REDUCIR EL RIESGO DE PARTO PREMATURO
}

\author{
Invertir y planificar
}

- Evaluar la necesidad de la oferta de los servicios de atención antes de la concepción y de oportunidades en el sistema de salud local para ser brindados.

- Utilizar todas las oportunidades para llegar a las niñas, mujeres y parejas, con mensajes preconcepcionales, que comienza en la escuela y que se extiende hasta los centros de salud y lugares comunitarios. La salud antes de la concepción también debe involucrar a los niños y hombres, para mejorar su salud y para involucrarlos en garantizar mejores resultados para las mujeres y las niñas.

\section{Implementar}

Aprovechar las oportunidades a través de los programas existentes (incluidos los programas no sanitarios) a:

- Educar a las mujeres y las parejas en edad reproductiva para tener un plan reproductivo que incluya la edad del primer embarazo, método para prevenir los embarazos no deseados y número de hijos que desean tener.

- Ampliar los programas de desarrollo personal y de desarrollo de habilidades, para negociar un comportamiento sexual seguro en la adolescencia. Adaptar intervenciones preconcepción para maximizar la adherencia por los adolescentes.

- Implementar la cobertura universal de vacunación infantil y de refuerzo de las enfermedades infecciosas que causan resultados adversos en el embarazo.

- Tamizar y tratar enfermedades infecciosas, particularmente infecciones de transmisión sexual.

- Fomentar una alimentación sana y el ejercicio para prevenir, tanto el bajo peso, como la obesidad en niñas y mujeres.

- Promover la seguridad alimentaria de las comunidades y los hogares. Ampliar los programas de nutrición para incluir niñas y adolescentes, especialmente para las mujeres con bajo peso, proporcionar suplementos de calorías de proteínas y micronutrientes. Una forma rentable de asegurar niveles adecuados de consumo de micronutrientes sería promulgar enriquecimiento a gran escala de alimentos de primera necesidad.

- Implementar políticas de salud pública para reducir el número de hombres y mujeres en edad reproductiva que usan tabaco.

- Implementar estrategias para el desarrollo comunitario y la reducción de la pobreza.

- Garantizar el acceso universal a la educación para empoderar a las niñas y mujeres con conocimientos básicos y habilidades que necesitan para tomar decisiones por sí mismas, como cuando acceder a la atención en salud.

\section{Aumentar proporcionalmente}

- Promover un método anticonceptivo eficaz para las mujeres/parejas, para espaciar los embarazos con 18 a 24 meses de diferencia.

- Tamización para condiciones crónicas, especialmente diabetes e hipertensión arterial, y referencia al centro de salud para su atención tan pronto como sea posible, para mejorar los resultados neonatales.

Informar y mejorar la cobertura y calidad de los programas

- Desarrollar indicadores para la vigilancia de la línea de base y para monitorear el progreso en la atención previa a la concepción.

- Incluir el parto prematuro entre los indicadores de seguimiento.

Innovar y emprender investigaciones aplicación

- Invertir en la investigación y el enlace a la acción. 


\section{Tabla VII \\ INTERVENCIONES PRIORITARIAS E INTERVENCIONES BASADAS EN LA EVIDENCIA DURANTE EL EMBARAZO, PARA REDUCIR LAS TASAS DE NACIMIENTOS PREMATUROS Y PARA BENEFICIAR AL NEONATO PREMATURO}

Los servicios prestados durante la atención prenatal

- Paquete básico de intervenciones para todas las mujeres embarazadas.

- Intervenciones situacionales (por ejemplo, identificación y tratamiento de la malaria, la tuberculosis y el VIH).

- Intervenciones adicionales como sea necesario (por ejemplo, de comportamiento, apoyo social e intervenciones financieras, intervenciones nutricionales que incluyen la administración de suplementos de calcio).

Atención de las mujeres embarazadas en mayor riesgo de parto prematuro, incluyendo

- Identificación y tratamiento de la enfermedad hipertensiva del embarazo.

- Monitoreo de los embarazos múltiples.

- Administración de progesterona para prolongar el embarazo.

- Identificación y tratamiento de las anomalías estructurales (insuficiencia de cuello uterino).

Atención de mujeres en trabajo de parto prematuro, incluyendo

- Tocolíticos para frenar el trabajo.

- Corticosteroides prenatales para reducir la mortalidad en los recién nacidos.

- Antibióticos para la ruptura prematura y prolongada de membranas para prevenir la infección neonatal.

- Suministro de sulfato de magnesio para la neuro-protección del recién nacido.

Intervenciones de la comunidad

- Promover la atención del parto prenatal calificado para todas las mujeres.

- Disminuir la exposición al tabaco.

Intervenciones de política

- Políticas de apoyo a la maternidad sin riesgos y acceso universal a la atención prenatal.

- Regular los horarios de trabajo y las condiciones laborales de la gestante.

- Establecer políticas profesionales y hospitalarias para regular los tratamientos de infertilidad y reducir las tasas de natalidad por cesárea y la inducción temprana del trabajo de parto. 


\title{
Tabla VIII ACCIONES DURANTE EL EMBARAZO PARA PREVENIR O GESTIONAR EL PARTO PREMATURO
}

\author{
Invertir y planificar
}

- Asegurar políticas nacionales y directrices existentes que proporcionen una adecuada protección de las mujeres embarazadas, con acceso universal a la atención integral prenatal, del parto, obstétrica de emergencia y atención postnatal.

- Asignar recursos suficientes para la prestación de la atención prenatal de alta calidad y equitativa, y la eliminación de las barreras a la atención, tales como pagos de los usuarios.

\section{Implementar}

- Aprovechar las oportunidades y los recursos, los enfoques y las oportunidades de formación de los programas existentes (incluidos los programas no sanitarios).

- Asegurar la existencia de un sistema de referencia funcional, sistemas de contratación justos, formación adecuada y supervisado, servicios de alta calidad para todas las mujeres embarazadas. Informar a las comunidades sobre la importancia de la atención prenatal, del parto y la atención postnatal para todas las mujeres, y los signos de alarma que incluyen el reconocimiento temprano de parto prematuro.

Informar y mejorar el programa en cuanto a cobertura y calidad

- Mejorar los registros y los datos, aumentar la supervisión y evaluación de programas para mejorar la calidad del servicio y el alcance a las poblaciones más pobres y vulnerables.

- Dar prioridad a la aplicación de la investigación, para promover aquellas intervenciones que resultan eficaces en diferentes contextos y en diferentes grupos de población.

Innovar y emprender investigaciones

- Invertir en investigación para el descubrimiento de la biología básica del embarazo normal y anormal, los determinantes genéticos de parto prematuro, y la investigación epidemiológica sobre factores de riesgo maternos, para proporcionar la evidencia necesaria para el desarrollo de estrategias eficaces de prevención y tratamiento.

\section{REFERENCIAS}

1. Garg A, Dworkin PH. Surveillance and screening for social determinants of health: the medical home and beyond. JAMA Pediatr. 2016;170(3):189-90.

2. Maness SB, Buhi ER. Associations between social determinants of health and pregnancy among young people. Public Health Rep. 2016;131(1):89-99.

3. International classification of diseases and related health problems. 10th revision. Geneva: World Health Organization; 1992.

4. Huddy CL, Johnson A, Hope PL. Educational and behavioral problems in babies of 32-35 weeks gestation. Arch Dis Child Fetal Neonatal 2001;85(1):F23-8.

5. Wang ML, Dorer DJ, Fleming MP, Catlin EA. Clinical outcomes of near-term infants. Pediatrics 2004;114:372-6.

6. Petrou S. The economic consequences of preterm birth during the first 10 years of life. BJOG 2005;112:105 .
7. Petrou S, Mehta Z, Hockley C, Cook-Mozaffari P, Henderson J, Goldacre M. The impact of preterm birth on hospital inpatient admissions and costs during the first 5 years of life. Pediatrics 2003;112:1290-7.

8. WHO. Recommended definitions, terminology and format for statistical tables related to the perinatal period and use of a new certificate for cause of perinatal deaths. Modifications recommended by FIGO as amended October 14, 1976. Acta Obstet Gynecol Scand 1977;56:247-53.

9. Beck S, Wojdyla D, Say L, Betran AP, Merialdi M, Requejo $\mathrm{JH}$, et al. The worldwide incidence of preterm birth: a systematic review of maternal mortality and morbidity. Bull World Health Organ 2010;88(1):31-8.

10. Blencowe $H$, Cousens $S$, Chou D, Oestergaard M, Say L, Moller AB, et al. Born Too Soon: The global epidemiology of 15 million preterm births. Reprod Health 2013;10(Suppl 1):S2.

11. March of Dimes, PMNCH, Save the Children, WHO. Born Too Soon: The Global Action Report on Preterm 
Birth. Eds. Howson CP, Kinney MV, Lawn JE. World Health Organization. Geneva, 2012.

12. Lalonde M. A new perspective on the health of Canadians. A working document. Ottawa: Government of Canada, 1974.

13. Villar M. Factores determinantes de la salud: Importancia de la prevención. Acta Med Per. 2011;28(4):237-41.

14. Martin JA, Hamilton BE, Menacker F, Sutton PD, Mathews TJ. Preliminary births for 2004: Infant and maternal health. Health e-stats. Released November 15, 2005. Acceso: 27-01-2016. Disponible en: http:// www.cdc.gov/nchs/products/pubs/pubd/hestats/prelimbirths04/prelimbirths04health.htm.

15. Blencowe $H$, Cousens $S$, Oestergaard MZ, Chou D, Moller AB, Narwal R, et al. Nacido Demasiado Pronto: Informe de Acción Global sobre Nacimientos Prematuros. Datos de estimaciones nacionales, regionales y mundiales de la tasa de nacimientos prematuros en el año 2010. Acceso: 28-01-2016. Disponible en: www. who.int/pmnch/media/news/2012/preterm_birth_report/es/index.html

16. PeriStats [online database]. White Plains, NY: March of Dimes; 2006. Acceso: 27-01-2016. Disponible en: http://www.marchofdimes.com/peristats/

17. Lawn JE, Wilczynska-Ketende K, Cousens SN. Estimating the causes of 4 million neonatal deaths in the year 2000. Int J Epidemiol 2006;35:706-18.

18. Liu L, Johnson H, Cousens S, Perin J, Scott S, Lawn $\mathrm{J}$, Ruden I, et al. Global, regional and national causes of child mortality: an updated systematic analysis for 2010 with time trends since 2000. Lancet 2012;379:2151-61.

19. Goldenberg RL, Jobe AH. Prospects for research in reproductive health and birth outcomes. JAMA 2001;285:633-9.

20. Kramer MS, Demissie K, Yang H, Platt RW, Sauve R, Liston $\mathrm{R}$. The contribution of mild and moderate preterm birth to infant mortality. Fetal and Infant Health Study Group of the Canadian Perinatal Surveillance System. JAMA 2000;284:843-9.

21. Lawn JE, Kerber K, Enweronu-Laryea C, Cousens S. 3.6 million neonatal deaths - what is progressing and what is not? Semin Perinatol 2010;34:371-86.

22. Slattery MM, Morrison JJ. Preterm delivery. Lancet 2002;360:1489-97.

23. Lawn JE, Cousens S, Zupan J. 4 million neonatal deaths: when? where? why? Lancet 2005;365:891-900.

24. Goldenberg RL, Gravett MG, lams J, Papageorghiou AT, Waller SA, Kramer M, et al. The preterm birth syndrome: issues to consider in creating a classification system. Am J Obstet Gynecol 2012;206:113-8.

25. Ananth CV, Joseph KS, Oyelese Y, Demissie K, Vintzileos AM. Trends in preterm birth and perinatal mortality among singletons: United States, 1989 through 2000. Obstet Gynecol 2005;105:1084-91.

26. Steer P. The epidemiology of preterm labour. BJOG 2005;112(Suppl 1):1-3.

27. Menon R. Spontaneous preterm birth, a clinical dile$\mathrm{mma}$ : etiologic, pathophysiologic and genetic heterogeneities and racial disparity. Acta Obstet Gynecol Scand 2008;87:590-600.

28. Plunkett J, Muglia LJ. Genetic contributions to preterm birth: implications from epidemiological and genetic association studies. Ann Med 2008;40:167-95.
29. Goldenberg RL, Culhane JF, lams JD, Romero R. Epidemiology and causes of preterm birth. Lancet 2008;371:75-84.

30. Muglia LJ, Katz M. The enigma of spontaneous preterm birth. N Engl J Med 2010;362:529-35.

31. Blondel B, Macfarlane A, Gissler M, Breart G, Zeitlin J. Preterm birth and multiple pregnancy in European countries participating in the PERISTAT project. BJOG 2006;113:528-35.

32. Felberbaum RE Multiple pregnancies after assisted reproduction - international comparison. Reprod Biomed Online 2007;15 (Suppl 3):53-60.

33. Simonsen KA, Anderson-Berry AL, Delair SF, Davies HD. Early-onset neonatal sepsis. Clin Microbiol Rev. 2014;27(1):21-47.

34. Chan GJ, Lee A, Baqui AH, Tan J, Black RE. Prevalence of early-onset neonatal infection among newborns of mothers with bacterial infection or colonization: a systematic review and meta-analysis. BMC Infec Dis. 2015; 15:118.

35. Polin RA. Management of neonates with suspected or proven early-onset bacterial sepsis. Pediatrics. 2012;129(5):1006-15.

36. Lee SE, Romero R, Park CW, Jun JK, Yoon BH. The frequency and significance of intraamniotic inflammation in patients with cervical insufficiency. Am J Obstet Gynecol. 2008;198(6):633.e1-8.

37. Gravett MG, Rubens CE, Nunes TM. Global report on preterm birth and stillbirth (2 of 7): discovery science. BMC Pregnancy Childbirth 2010;10 (Suppl 1):S2.

38. Mendonca AS, Figueiredo AE, Oliveira LM, Ferreira F, Pinheiro V, Fróes K, et al. Association between chronic apical periodontitis and low-birth-weight preterm births. J Endodontics. 2015;41(3):353-7.

39. Ananth CV, Vintzileos AM. Epidemiology of preterm birth and its clinical subtypes. J Matern Fetal Neonatal Med 2006;19:773-82.

40. Zeitlin J, Saurel-Cubizolles MJ, De Mouzon J, Rivera L, Ancel PY, Blondel B, Kaminski M. Fetal sex and preterm birth: are males at greater risk? Hum Reprod 2002;17:2762-8.

41. Barros FC, Papageorghiou AT, Victora CG, Noble JA, Pang R, lams J, et al. The distribution of clinical phenotypes of preterm birth syndrome implications for prevention. JAMA Pediatr. 2015;169(3):220-9.

42. Patel RR, Steer P, Doyle P, Little MP, Elliott P. Does gestation vary by ethnic group? A London-based study of over 122,000 pregnancies with spontaneous onset of labour. Int J Epidemiol. 2004;33:107-13.

43. Kalra SK, Molinaro TA. The association of in vitro fertilization and perinatal morbidity. Semin Reprod Med. 2008;26:423-35

44. Park K (ed). Health care of the community. In: Textbook of Preventive and Social Medicine. 20th ed. Jabalpur: Banarsidas Bhanot Publishers; 2009; 7934.

45. WHO. State of Inequality: Reproductive, Maternal, Newborn and Child Health. Geneva: WHO Press; 2015.

46. Diderichsen F, Andersen I, Manuel C; Working Group of Danish Review on Social Determinants of Health, Andersen AM, Bach E, Baadsgaard M, et al. Health inequality - Determinants and policies. Scand J Public Health 2012;40(Suppl 8):12 105. 
47. Chen L, Wu Y, Coyte PC. Income related children's health inequality and health achievement in China. Int J Equity Health 2014;13:102.

48. Ataguba JE, Akazili J, Mclntyre D. Socioeconomic related health inequality in South Africa: Evidence from General Household Surveys. Int J Equity Health 2011;10:48.

49. McCall SJ, Bhattacharya S, Okpo E, Macfarlane GJ. Evaluating the social determinants of teenage pregnancy: a temporal analysis using a UK obstetric database from 1950 to 2010. J Epidemiol Community Health 2015;69:49-54.

50. Rodriguez $\mathrm{Ml}$, Chang $\mathrm{R}$, Thiel de Bocanegra $\mathrm{H}$. The impact of postpartum contraception on reducing preterm birth: findings from California. Am J Obstet Gynecol 2015;213:703.e1-6.

51. WHO. Department of Reproductive Health and Research. Randomized Trial WHO antenatal care: a manual for the implementation of the new antenatal care model; 2003. Disponible en: http://whqlibdoc.who.int/ hq/2001/WHO_RHR_01.30.pdf. Acceso: 25/2/2012.

52. Antenatal care routine care for healthy pregnant woman. National Collaborating Centre for Women's and Children's Health. National Institute for Health and Clinical Excellence. NICE Clinical Guideline 62;2008. Disponible en: http://www.nice.org.uk/nicemedia/pdf/ CG62fullguideline.pdf. Acceso: 11/9/2012.

53. Villar J, Carroli G, Khan-Neelofur D, Piaggio G, Gulmezoglu M. Patterns of routine antenatal care for low-risk pregnancy. Cochrane Database Syst Rev. 2001;(4):CD000934. Acceso: 29-012016. Disponible en: http://onlinelibrary.wiley.com/ doi/10.1002/14651858.CD000934/pdf

54. Villar J, Ba'aqeel H, Piaggio G, Lumbiganon P, Miguel Belizan J, Farnot U, et al. WHO antenatal care randomised trial for the evaluation of a new model of routine antenatal care. Lancet. 2001;357:1551-64.

55. Paz-Zulueta M, Llorca J, Sarabia-Lavín R, Bolumar F, Rioja L, Delgado A, et al. The role of prenatal care and social risk factors in the relationship between immigrant status and neonatal morbidity: a retrospective cohort study. PLOS ONE 10(3):e0120765.
56. Mendoza LA, Arias MD, Peñaranda CB, Mendoza LI, Manzano S, Varela AM. Influencia de la adolescencia y su entorno en la adherencia al control prenatal e impacto sobre la prematuridad, bajo peso al nacer y mortalidad neonatal. Rev Chil Obstet Ginecol. 2015;80(4):306-15.

57. Redding S, Conrey E, Porter K, Paulson J, Hughes K, Redding M. Pathways Community Care Coordination in Low Birth Weight Prevention. Maternal Child Health J 2015;19(3):643-50.

58. Reijneveld SA. Reported health lifestyles and use of health care of first generation immigrants in The Netherlands: do socioeconomic factors explain their adverse position?. J Epidemiol Community Health. 1998;52:298-304.

59. Dunn JR, Dyck I. Social determinants of health in Canada's immigrant population: results from the National Population Health Survey. Soc Sci Med. 2000;51:1573-93.

60. Borrell C, Muntaner C, Sola J, Artazcoz L, Puigpinos R, Benach J, et al. Immigration and self-reported health status by social class and gender: the importance of material deprivation, work organisation and household labour. J Epidemiol Community Health. 2008;62(5):e7.

61. Malmusi D, Borrell C and Benach J. Migration-related health inequalities: showing the complex interactions between gender, social class and place of origin. Soc Sci Med. 2010;71:1610-9.

62. Rodriguez C, Regidor E, Gutierrez-Fisac JL. Low birth weight in Spain associated with sociodemographic factors. J Epidemiol Community Health. 1995;49:3842.

63. Sparks PJ. Do biological, sociodemographic, and behavioral characteristics explain racial/ethnic disparities in preterm births? Soc Sci Med. 2009;68:1667-75.

64. Garcia-Subirats I, Perez G, Rodriguez-Sanz M, Salvador J, Jane M. Recent immigration and adverse pregnancy outcomes in an urban setting in Spain. Matern Child Health J. 2011;15:561-9. 